\title{
Effects of the Covid-19 on the Marine industry: Evidence from the ten major marine companies
}

\author{
Alexandros Pasiouras \& Theodoros Daglis \\ National Technical Univeristy of Athens
}

\begin{abstract}
:
The Covid-19 pandemic has caused many economic problem on the Marine industry. More precisely, the lockdown measures, the total suspension of the Marine activity and the disruption of the Marine field have led to the severe impact on the Marine corporations globally. In this paper, we examine some of the biggest Marine companies' stocks, namely the Dynangas (DLNG), Euronav (EURN), Euroseas (ESEA), Frontline (FRO), Nordic American Tanker (NAT), Scorpio Bulkers (SALT), Sino-Global Shipping (SINO), Teekay Tankers (TNK), Tsakos Energy Navigation (TNP), Torm (TRMD) during the Covid-19 spread and the lockdown measures. Using relevant time series specifications, we establish a Bayesian framework to unveil the effect of the Covid-19 pandemic on these stocks. Based on our findings, the stocks were affected by the Covid19 spread and more precisely, the Covid-19 spread provides useful information for the predicting and forecasting of these stocks. Our findings are robust, since the out-ofsample forecasting accuracy of the alternative model employed, that explicitly incorporate the pandemic induced by the SARS-COV-2 virus, is superior to the baseline model.
\end{abstract}

Keywords: COVID-19, maritime, stocks

JEL codes: C22, C58, C50, C51

\section{Introduction}

The recent Covid-19 pandemic has spread from one country to another, having its origin in Wuhan-Hubei, China (Liu et al., 2020). The total cases globally amount to over 2 million people, showing the cruel face of this pandemic (WHO, 2020). The case of COVID-19 is unique, differing in many ways from previous disease spreads, such as the severe acute respiratory syndrome (SARS) spread in 2003 (Wilder-Smith et al., 2020). The exponential spread of the SARS-COV-2 virus in a worldwide context has spread fear globally. 
In an attempt to minimize the spread of the virus, most economies and policy makers have taken extreme lockdown measures that adversely affect the overall microeconomic, macroeconomic and financial conditions in a global scale. Increased globalization and integration, of trade and financial relations, among the various economies and institutions, makes clear that the global cost of the pandemic will not be limited to the directly affected economies, since the indirect spillover effects are of great importance, as well (Lee and McKibbin, 2004).

Such cases are the marine companies that were affected both by the lockdown measures, due to the suspension of their proper function and by the economic recession followed by the Covid-19 spread. The lockdown measures had a very important and negative effect on the marine industry. More analytically, Depellegrina et al. (2020) argue that vessel activity were reduced by $69 \%$ during the lockdown measures of the Covid-19, fishing activities reduced by $84 \%$ and passenger traffic by $78 \%$.

In this paper, using Bayesian techniques with a novel approach, we capture the impact of the COVID-19 spread on the biggest marine companies and more precisely on the Dynangas (DLNG), Euronav (EURN), Euroseas (ESEA), Frontline (FRO), Nordic American Tanker (NAT), Scorpio Bulkers (SALT), Sino-Global Shipping (SINO), Teekay Tankers (TNK), Tsakos Energy Navigation (TNP), Torm (TRMD) stocks.

The present paper contributes to the literature in the following ways: (a) It is the first, to the best of our knowledge, that investigates the effect of the Covid-19 spread and the lockdown measures on the major marine companies through the financial system; (b) It is among the first, to the best of our knowledge, that accounts for the Covid-19 pandemic in a financial framework, using global data on the spread of the pandemic; (c) it proposes an alternative approach to the examination of the economic effect of disasters on the marine companies, based on a financial framework; (d) it provides a novel Bayesian framework and (e) it provides a robustness analysis of the findings based on out-of-sample forecasting accuracy measures.

The paper is structured as follows: Section 2 sets out the the methodology used, Section 3 presents the empirical results and finally, Section 4 concludes the paper.

\section{Methodology}

Following standard time series literature (Wooldridge 2013), we examine the level of integration of the time series that enter our analysis using the Phillips-Perron (1988) unit root test. More specifically, the hypothesis tested for the Phillips - Perron test is that the time series does not have a unit root. In addition, in case of integrated of degree one time series, i.e. I(1), we also investigate the potential existence of long-run relationships among the variables, using the popular Johansen (1990) cointegration test, and the 
2nd International Conference on Applied Research in BUSINESS, MANAGEMENT and ECONOMICS
25-27 September 2020

Berlin, Germany

hypothesis tested is that the time series are not cointegrated. Finally, the optimal lag length of the time series variables was investigated using the Schwartz-Bayes information criterion (SBIC).

In order to cross validate the fact that the Covid-19 confirmed cases have predictive ability on the major marine companies' stocks, we will make use of forecasting strategies. In detail, using a vector autoregressive model in case of no-cointegration, or a vector error correction model in case of integration, as a baseline model, we will investigate whether alternative specification of the Bayesian model outperforms the forecasting accuracy of the baseline model. We should note that in the models is incorporate the information provided by Covid-19 confirmed cases as an exogenous variable. To compare the models, three distinct measure of forecasting accuracy are used, namely the mean absolute error (MAE), the mean absolute percentage error (MAPE) and the root mean square forecasting error (RMSFE), to investigate the magnitude of the predictive power of Covid-19 spread on the biggest marine companies' stocks. this work.

In what follows, we offer a brief outline of the techniques and procedures used in

\subsection{Econometric models}

A basic and classical econometric approach is the vector autoregressive model - VAR, or the vector error correction model - VECM in presence of co-integration relationship among the variables.

\section{VEC Model:}

In presence of co-integration relationship among the variables, a physical extension of the VAR model is the VEC model. In this case, we incorporate the error correction term. The model is structured as follows:

Again, each variable in the model, has an equation explaining its evolution based on its own lagged values, the lagged values of the other model variables, the error correction, and an error term. A VECM model of order $\mathrm{p}$, with exogenous variables is structured as follows:

$$
\left\{\begin{array}{c}
\Delta y_{1, t}=c_{1}+\sum_{j}^{n} \sum_{i}^{p}\left(a_{j, i} \Delta y_{j, t-i}\right)+\sum_{j}^{k} \sum_{i}^{q}\left(b_{j, i} \Delta x_{j, t-i}\right)+v_{1, t}+e_{1, t} \\
\Delta y_{2, t}=c_{2}+\sum_{j}^{n} \sum_{i}^{p}\left(a_{j, i} \Delta y_{j, t-i}\right)+\sum_{j}^{k} \sum_{i}^{q}\left(b_{j, i} \Delta x_{j, t-i}\right)+v_{2, t}+e_{2, t} \\
\quad \cdots \\
\Delta y_{n, t}=c_{n}+\sum_{j}^{n} \sum_{i}^{p}\left(a_{j, i} \Delta y_{j, t-i}\right)+\sum_{j}^{k} \sum_{i}^{q}\left(b_{j, i} \Delta x_{j, t-i}\right)+v_{n, t}+e_{n, t}
\end{array}\right\}
$$

Again, $\mathrm{n}$ is the number of the dependent variables $\left(y_{i, t}\right)$ of the model, $c_{i}$ is the fixed term, $\mathrm{p}$ is the lag order of the dependent variables, $e_{i, t}$ is the error term of each equation of the model, and $v_{i, t}$ is the error correction term, as before. In the case of 
exogenous variables, $\mathrm{k}$ is the number of the independent or exogenous variables $\left(x_{i, t}\right)$ of the model and $\mathrm{q}$ is the lag order of the exogenous variables.

\subsection{Bayesian model}

The idea of our model is that we use in the first step simple linear regression in order to get some initial estimations for the maritime stocks. Next, we use a Bayesian dynamics model to improve the estimations of the previous step and eventually calibrate the data curve, so that we are able to make satisfactory forecasts.

To begin with, we regress the exogenous variable to itself with lag 1 and each of the maritime stock to itself again with lag 1.

$\widehat{x}_{\iota}=\lambda_{0}^{(j)}+\lambda_{1}^{(j)} \widehat{x}_{\iota}+w_{i j}, w_{i j} \sim N\left(0, \sigma_{1}^{2}\right)$

${\widehat{y_{l}}}^{(j)}=\theta_{0}^{(j)}+\theta_{1}^{(j)} \widehat{y_{l-1}}{ }^{(j)}+u_{i j}, u_{i j} \sim N\left(0, \sigma_{2}^{2}\right)$

Then we use these estimations within the new model

$$
y_{i}^{(j)}=\mu_{j}+\sum_{k=1}^{m} a_{j k} y_{i-1}^{(k)}+b_{j} x_{i-1}+c_{j} \widehat{x}_{\imath}+p_{j} \widehat{y_{l}^{(\jmath)}}+q_{j}\left(\widehat{y_{l}^{(J)}}-\widehat{y_{l-1}^{(\jmath)}}\right)+\epsilon_{i}^{(j)},
$$

$j=1,2, \cdots, m$, for each $1 \leq i \leq N$.

We suppose that for each $j=1, \cdots m, \epsilon_{i}=\left(\epsilon_{i}^{(1)}, \cdots, \epsilon_{i}^{(m)}\right) \sim N_{m}(0, \Sigma)$

The parameters $\left(\mu_{1}, \cdots, \mu_{m}\right),\left(b_{1}, \cdots, b_{m}\right),\left(c_{1}, \cdots, c_{m}\right),\left(p_{1}, \cdots, p_{m}\right),\left(q_{1}, \cdots, q_{m}\right), A \in$ $\mathbb{R}^{m \times m}, \Sigma \in \mathbb{R}^{m \times m}$ are estimated through Bayesian framework using Gibbs algorithm by working the multivariate normal and inverse Wishart distribution as the conjugate of the prior distribution selections.

3.Result Analysis

3.1Data and variables

The data used in the present paper are the global Confirmed cases of the Covid-19 in daily format and were downloaded by the John Hopkins University database and span the period 22 January 2020 until 29 July 2020. Moreover, in the present paper we used financial stocks of some of the biggest Cruise companies, namely the Dynangas (DLNG), 
Euronav (EURN), Euroseas (ESEA), Frontline (FRO), Nordic American Tanker (NAT), Scorpio Bulkers (SALT), Sino-Global Shipping (SINO), Teekay Tankers (TNK), Tsakos Energy Navigation (TNP), Torm (TRMD) as derived from finance.yahoo, and span also the period 22 January 2020 until 29 July 2020. The timeseries were transformed into logarithmic scale. The descriptive statistics of the timeseries are depicted in Table 1. Furthermore, the plots of the logarithmic timeseries are depicted on Figure 1.

Table 1: Descriptive statistics of the logarithmic timeseries.

\begin{tabular}{ccccc}
\hline Timeseries & Mean & Sd & Min & Max \\
\hline DLNG & 0.280 & 0.166 & 0.004 & 0.589 \\
ESEA & 0.403 & 0.068 & 0.238 & 0.576 \\
EURN & 0.942 & 0.033 & 0.857 & 1.025 \\
FRO & 0.888 & 0.051 & 0.770 & 1.025 \\
NAT & 0.596 & 0.100 & 0.378 & 0.853 \\
SALT & 1.338 & 0.143 & 1.127 & 1.687 \\
SINO & 0.326 & 0.075 & 0.190 & 0.591 \\
TNK & 1.218 & 0.075 & 1.090 & 1.401 \\
TNP & 1.104 & 0.090 & 0.932 & 1.316 \\
TRMD & 0.885 & 0.039 & 0.815 & 1.097 \\
Confirmed & 6.021 & 1.082 & 2.744 & 7.231 \\
\hline
\end{tabular}

Figure 1: Logarithmic timeseries plots.

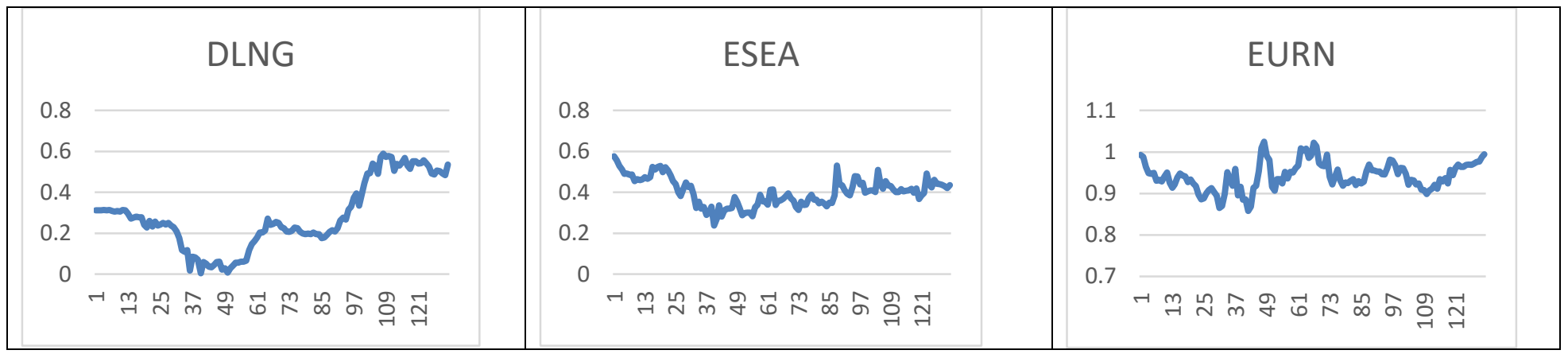




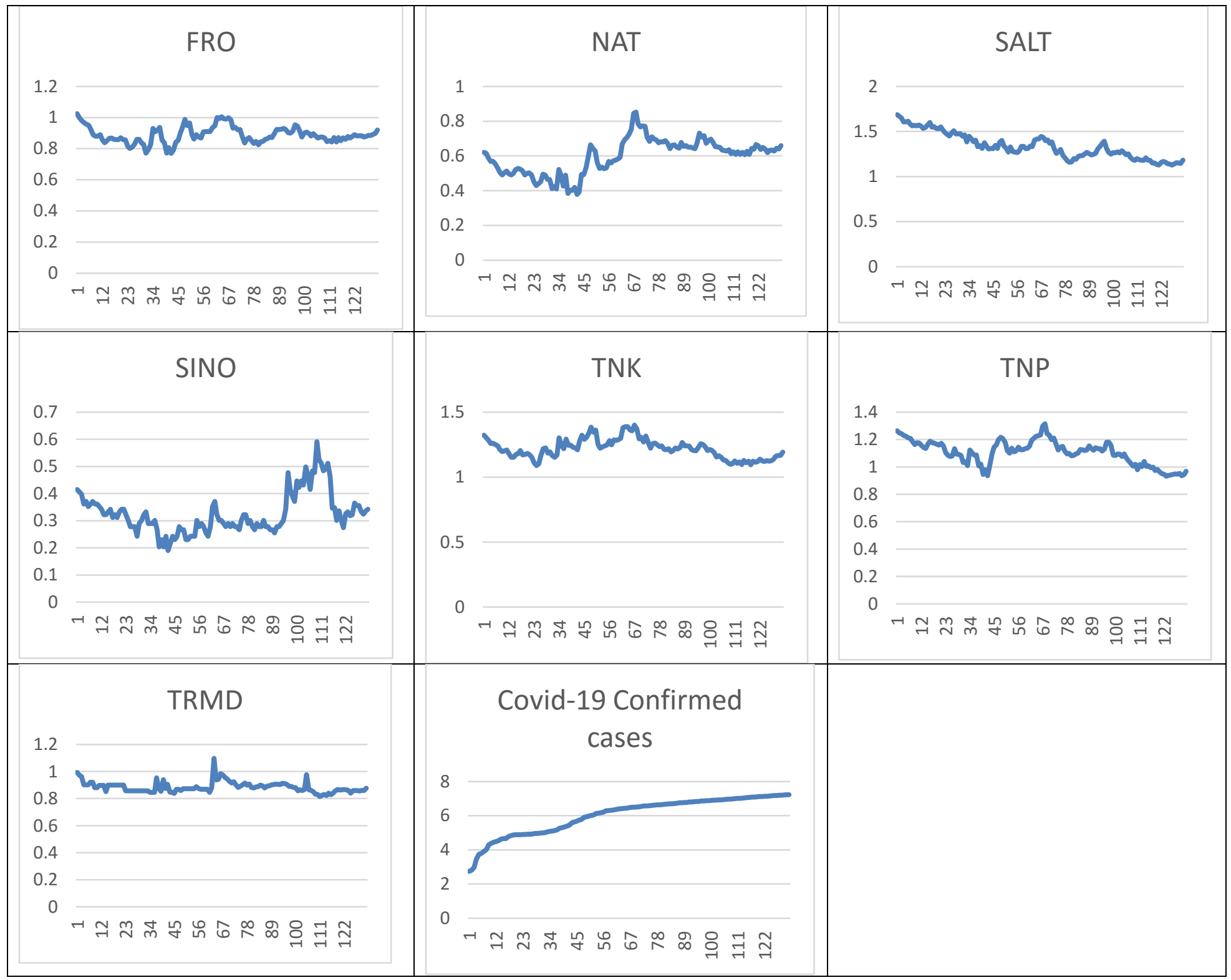

\subsection{Results}

In the present paper, we set out a hypothesis tested, that the Covid-19 spread, and more analytically, the current economic crisis and the aftermath of the lockdown measures following the Covid-19, affect the trading of the major marine companies' stocks.

In the present work, we test the contribution of the information derived by the Covid-19 confirmed cases on the forecasting of the aforementioned stocks. To do so, we make use of two econometric models in the form of vector autoregressive model (since there is connection among the three stocks). The first model, declared as the baseline, 
will be a vector autoregressive model and an alternative model being the Bayesian model. Both models have one exogenous variable, the Covid-19 global confirmed cases.

A first step in every econometric modeling is the unit root test. In the present paper, we make use of the Phillips - Perron unit root test. The results are depicted in Table 2.

Table 2: Phillips - Perron unit root test results.

\begin{tabular}{ccc}
\hline Timeseries & P-val of PP-test & Order of Integration \\
\hline DLNG & 0.736 & $\mathrm{I}(1)$ \\
ESEA & 0.095 & $\mathrm{I}(0)$ \\
EURN & 0.015 & $\mathrm{I}(0)$ \\
FRO & 0.01913 & $\mathrm{I}(0)$ \\
NAT & 0.321 & $\mathrm{I}(1)$ \\
SALT & 0.09718 & $\mathrm{I}(0)$ \\
SINO & 0.2146 & $\mathrm{I}(1)$ \\
TNK & 0.3635 & $\mathrm{I}(1)$ \\
TNP & 0.3305 & $\mathrm{I}(1)$ \\
TRMD & 0.01 & $\mathrm{I}(0)$ \\
Confirmed & 0.01 & $\mathrm{I}(0)$ \\
\hline
\end{tabular}

Since the results in Table 2 show that some timeseries have no unit root, meaning that the timeseries are stationary, while others have unit root, meaning that they are not stationary, a cointegration test is the next step of analysis.

Table 3: Johansen Cointegration test results.

\begin{tabular}{ccccc}
\hline Rank of co-integration & Co-integration test & 10pct & 5pct & 1pct \\
\hline $\mathrm{r}<=10$ & 0.430 & 6.500 & 8.180 & 11.650 \\
$\mathrm{r}<=9$ & 5.260 & 12.910 & 14.900 & 19.190 \\
$\mathrm{r}<=8$ & 8.900 & 18.900 & 21.070 & 25.750 \\
$\mathrm{r}<=7$ & 11.070 & 24.780 & 27.140 & 32.140 \\
$\mathrm{r}<=6$ & 23.130 & 30.840 & 33.320 & 38.780 \\
$\mathrm{r}<=5$ & 27.540 & 36.250 & 39.430 & 44.590 \\
$\mathrm{r}<=4$ & 32.340 & 42.060 & 44.910 & 51.300 \\
$\mathrm{r}<=3$ & 42.810 & 48.430 & 51.070 & 57.070 \\
$\mathrm{r}<=2$ & 52.200 & 54.010 & 57.000 & 63.370 \\
$\mathrm{r}<=1$ & 71.820 & 59.000 & 62.420 & 68.610 \\
$\mathrm{r}=0$ & 91.060 & 65.070 & 68.270 & 74.360 \\
\hline
\end{tabular}


The results of the co-integration test are depicted in Table 3, indicate that there are 2 cointegration relationships among the timeseries. In such case, an error correction term must be included in the econometric models.

We will next test if the Bayesian model, proposed in the present paper, is better in terms of forecasting ability than the baseline econometric model. To do so, we first decide for the lag order of the econometric model, based on the SBIC criterion. The results are depicted in Table 4.

Table 4: Results of the SBIC criterion for the case of the Baseline and Alternative models.

\begin{tabular}{ccccc}
\hline Lag Order & AIC(n) & HQ(n) & SC(n) & FPE(n) \\
\hline 1 & -66.265 & -65.033 & -63.232 & $1.676 \mathrm{E}-29$ \\
2 & -65.636 & -63.274 & -59.821 & $3.285 \mathrm{E}-29$ \\
3 & -65.391 & -61.899 & -56.795 & $4.716 \mathrm{E}-29$ \\
4 & -65.038 & -60.417 & -53.661 & $8.489 \mathrm{E}-29$ \\
5 & -65.471 & -59.721 & -51.313 & $8.323 \mathrm{E}-29$ \\
6 & -66.432 & -59.551 & -49.493 & $6.296 \mathrm{E}-29$ \\
7 & -67.453 & -59.443 & -47.733 & $6.766 \mathrm{E}-29$ \\
8 & -69.653 & -60.514 & -47.152 & $4.4 \mathrm{E}-29$ \\
9 & -74.428 & -64.160 & -49.146 & $7.724 \mathrm{E}-30$ \\
10 & -94.406 & -83.007 & -66.342 & $7.812 \mathrm{E}-36$ \\
\hline
\end{tabular}

Based on Table 4, all the criterions indicate the lag order 10 as the most appropriate for both models. In this case, the order of the baseline econometric order will be selected to be equal to 10 .

Next, using out of sample forecast with fixed window, for horizon $\mathrm{H}=1,2, \ldots, 20$, we forecast for approximately two months. Then, we compare the two models in terms of forecasting ability, based on the MAE, MAPE, RMSFE. The results are depicted in Tables 5-14.

Table 5: MAE, MAPE and RSMFE forecasting accuracy of the Bayesian and VECX models for the case of DLNG.

\begin{tabular}{rrrrrr}
\hline Bayes_MAE & Bayes_MAPE & Bayes_RMSFE & VECX_MAE & VECX_MAPE & VECX_RMSFE \\
\hline 0.014 & 0.011 & 0.014 & 0.120 & 0.093 & 0.120 \\
0.018 & 0.015 & 0.019 & 0.178 & 0.141 & 0.188 \\
0.034 & 0.028 & 0.041 & 0.189 & 0.152 & 0.196 \\
0.068 & 0.058 & 0.092 & 0.152 & 0.123 & 0.171 \\
0.081 & 0.071 & 0.102 & 0.128 & 0.104 & 0.153 \\
0.084 & 0.073 & 0.101 & 0.123 & 0.101 & 0.146
\end{tabular}


2nd International Conference on Applied Research in BUSINESS, MANAGEMENT and ECONOMICS
25-27 September 2020

Berlin, Germany

\begin{tabular}{llllll}
0.075 & 0.065 & 0.094 & 0.124 & 0.102 & 0.143 \\
0.080 & 0.070 & 0.097 & 0.138 & 0.115 & 0.158 \\
0.131 & 0.115 & 0.201 & 0.148 & 0.126 & 0.168 \\
0.130 & 0.114 & 0.195 & 0.168 & 0.141 & 0.193 \\
\hline
\end{tabular}

Table 6: MAE, MAPE and RSMFE forecasting accuracy of the Bayesian and VECX models for the case of ESEA.

\begin{tabular}{rrrrrr}
\hline Bayes_MAE & Bayes_MAPE & Bayes_RMSFE & VECX_MAE & VECX_MAPE & VECX_RMSFE \\
\hline 0.189 & 0.166 & 0.189 & 0.284 & 0.251 & 0.284 \\
0.130 & 0.118 & 0.142 & 0.260 & 0.242 & 0.262 \\
0.099 & 0.092 & 0.118 & 0.213 & 0.201 & 0.224 \\
0.104 & 0.097 & 0.119 & 0.254 & 0.240 & 0.271 \\
0.102 & 0.095 & 0.114 & 0.324 & 0.311 & 0.363 \\
0.104 & 0.098 & 0.114 & 0.379 & 0.366 & 0.426 \\
0.100 & 0.096 & 0.110 & 0.436 & 0.424 & 0.491 \\
0.120 & 0.116 & 0.136 & 0.486 & 0.477 & 0.547 \\
0.145 & 0.143 & 0.174 & 0.568 & 0.564 & 0.657 \\
0.232 & 0.230 & 0.360 & 0.676 & 0.671 & 0.813 \\
\hline
\end{tabular}

Table 7: MAE, MAPE and RSMFE forecasting accuracy of the Bayesian and VECX models for the case of EURN.

\begin{tabular}{rrrrrr}
\hline Bayes_MAE & Bayes_MAPE & Bayes_RMSFE & VECX_MAE & VECX_MAPE & VECX_RMSFE \\
\hline 0.023 & 0.010 & 0.023 & 0.249 & 0.112 & 0.249 \\
0.017 & 0.007 & 0.018 & 0.356 & 0.161 & 0.372 \\
0.012 & 0.006 & 0.015 & 0.409 & 0.184 & 0.424 \\
0.010 & 0.004 & 0.013 & 0.480 & 0.216 & 0.506 \\
0.011 & 0.005 & 0.013 & 0.588 & 0.264 & 0.642 \\
0.019 & 0.009 & 0.028 & 0.698 & 0.313 & 0.776 \\
0.039 & 0.017 & 0.065 & 0.824 & 0.368 & 0.934 \\
0.068 & 0.030 & 0.113 & 0.958 & 0.428 & 1.102 \\
0.071 & 0.031 & 0.111 & 1.111 & 0.495 & 1.299 \\
0.143 & 0.063 & 0.272 & 1.293 & 0.573 & 1.542 \\
\hline
\end{tabular}

Table 8: MAE, MAPE and RSMFE forecasting accuracy of the Bayesian and VECX models for the case of FRO. 


\begin{tabular}{rrrrrr}
\hline Bayes_MAE & Bayes_MAPE & Bayes_RMSFE & VECX_MAE & VECX_MAPE & VECX_RMSFE \\
\hline 0.086 & 0.042 & 0.086 & 0.280 & 0.138 & 0.280 \\
0.082 & 0.040 & 0.082 & 0.413 & 0.203 & 0.434 \\
0.080 & 0.039 & 0.080 & 0.517 & 0.254 & 0.548 \\
0.081 & 0.040 & 0.081 & 0.640 & 0.316 & 0.693 \\
0.082 & 0.041 & 0.082 & 0.776 & 0.383 & 0.856 \\
0.079 & 0.039 & 0.079 & 0.914 & 0.450 & 1.019 \\
0.076 & 0.037 & 0.076 & 1.069 & 0.526 & 1.210 \\
0.083 & 0.041 & 0.086 & 1.233 & 0.605 & 1.409 \\
0.085 & 0.042 & 0.088 & 1.422 & 0.696 & 1.651 \\
0.150 & 0.072 & 0.247 & 1.638 & 0.795 & 1.933 \\
\hline
\end{tabular}

Table 9: MAE, MAPE and RSMFE forecasting accuracy of the Bayesian and VECX models for the case of NAT.

\begin{tabular}{rrrrrr}
\hline Bayes_MAE & Bayes_MAPE & Bayes_RMSFE & VECX_MAE & VECX_MAPE & VECX_RMSFE \\
\hline 0.069 & 0.047 & 0.069 & 0.184 & 0.126 & 0.184 \\
0.052 & 0.035 & 0.055 & 0.377 & 0.253 & 0.423 \\
0.049 & 0.033 & 0.051 & 0.471 & 0.318 & 0.514 \\
0.063 & 0.043 & 0.069 & 0.573 & 0.392 & 0.625 \\
0.068 & 0.047 & 0.073 & 0.711 & 0.488 & 0.795 \\
0.067 & 0.046 & 0.072 & 0.854 & 0.586 & 0.969 \\
0.058 & 0.040 & 0.066 & 1.027 & 0.706 & 1.189 \\
0.078 & 0.053 & 0.098 & 1.228 & 0.840 & 1.451 \\
0.070 & 0.048 & 0.093 & 1.462 & 0.997 & 1.761 \\
0.090 & 0.061 & 0.123 & 1.738 & 1.176 & 2.140 \\
\hline
\end{tabular}

Table 10: MAE, MAPE and RSMFE forecasting accuracy of the Bayesian and VECX models for the case of SALT.

\begin{tabular}{rrrrrr}
\hline Bayes_MAE & Bayes_MAPE & Bayes_RMSFE & VECX_MAE & VECX_MAPE & VECX_RMSFE \\
\hline 0.003 & 0.001 & 0.003 & 0.109 & 0.040 & 0.109 \\
0.004 & 0.002 & 0.004 & 0.062 & 0.023 & 0.078 \\
0.013 & 0.005 & 0.018 & 0.046 & 0.017 & 0.064 \\
0.016 & 0.006 & 0.020 & 0.059 & 0.022 & 0.074 \\
0.024 & 0.009 & 0.030 & 0.058 & 0.022 & 0.071 \\
0.026 & 0.010 & 0.031 & 0.056 & 0.021 & 0.067 \\
0.028 & 0.011 & 0.033 & 0.054 & 0.020 & 0.064
\end{tabular}


2nd International Conference on Applied Research in BUSINESS, MANAGEMENT and ECONOMICS
25-27 September 2020

Berlin, Germany
0.031
0.012
0.035
0.049
0.019
0.060
0.109
0.041
0.247
0.045
0.017
0.057
0.160
0.060
0.306
0.044
0.017
0.055

Table 11: MAE, MAPE and RSMFE forecasting accuracy of the Bayesian and VECX models for the case of SINO.

\begin{tabular}{rrrrrr}
\hline Bayes_MAE & Bayes_MAPE & Bayes_RMSFE & VECX_MAE & VECX_MAPE & VECX_RMSFE \\
\hline 0.012 & 0.016 & 0.012 & 0.239 & 0.312 & 0.239 \\
0.015 & 0.020 & 0.015 & 0.333 & 0.448 & 0.347 \\
0.016 & 0.021 & 0.016 & 0.409 & 0.551 & 0.430 \\
0.034 & 0.043 & 0.047 & 0.530 & 0.678 & 0.581 \\
0.045 & 0.056 & 0.058 & 0.694 & 0.871 & 0.796 \\
0.056 & 0.069 & 0.069 & 0.894 & 1.112 & 1.062 \\
0.065 & 0.081 & 0.078 & 1.128 & 1.423 & 1.373 \\
0.068 & 0.085 & 0.079 & 1.361 & 1.745 & 1.663 \\
0.098 & 0.125 & 0.135 & 1.618 & 2.082 & 1.990 \\
0.161 & 0.205 & 0.265 & 1.935 & 2.481 & 2.420 \\
\hline
\end{tabular}

Table 12: MAE, MAPE and RSMFE forecasting accuracy of the Bayesian and VECX models for the case of TNK.

\begin{tabular}{rrrrrr}
\hline Bayes_MAE & Bayes_MAPE & Bayes_RMSFE & VECX_MAE & VECX_MAPE & VECX_RMSFE \\
\hline 0.089 & 0.034 & 0.089 & 0.290 & 0.112 & 0.290 \\
0.088 & 0.034 & 0.088 & 0.470 & 0.182 & 0.503 \\
0.083 & 0.032 & 0.083 & 0.591 & 0.228 & 0.632 \\
0.079 & 0.031 & 0.079 & 0.709 & 0.274 & 0.763 \\
0.075 & 0.029 & 0.076 & 0.863 & 0.333 & 0.951 \\
0.064 & 0.025 & 0.069 & 1.027 & 0.395 & 1.150 \\
0.063 & 0.024 & 0.068 & 1.211 & 0.463 & 1.378 \\
0.089 & 0.034 & 0.116 & 1.422 & 0.539 & 1.646 \\
0.081 & 0.031 & 0.109 & 1.662 & 0.628 & 1.959 \\
0.075 & 0.028 & 0.104 & 1.939 & 0.726 & 2.328 \\
\hline
\end{tabular}

Table 13: MAE, MAPE and RSMFE forecasting accuracy of the Bayesian and VECX models for the case of TNP.

Bayes_MAE $\quad$ Bayes_MAPE $\quad$ Bayes_RMSFE $\quad$ VECX_MAE $\quad$ VECX_MAPE $\quad$ VECX_RMSFE 


\begin{tabular}{llllll}
\hline 0.061 & 0.029 & 0.061 & 0.139 & 0.065 & 0.139 \\
0.052 & 0.024 & 0.053 & 0.257 & 0.119 & 0.282 \\
0.043 & 0.020 & 0.046 & 0.303 & 0.140 & 0.324 \\
0.034 & 0.016 & 0.040 & 0.353 & 0.163 & 0.378 \\
0.036 & 0.017 & 0.040 & 0.405 & 0.187 & 0.435 \\
0.036 & 0.017 & 0.040 & 0.458 & 0.211 & 0.495 \\
0.042 & 0.019 & 0.047 & 0.520 & 0.239 & 0.568 \\
0.081 & 0.038 & 0.134 & 0.586 & 0.270 & 0.649 \\
0.086 & 0.040 & 0.132 & 0.669 & 0.308 & 0.756 \\
0.095 & 0.044 & 0.138 & 0.764 & 0.350 & 0.881 \\
\hline
\end{tabular}

Table 14: MAE, MAPE and RSMFE forecasting accuracy of the Bayesian and VECX models for the case of TRMD.

\begin{tabular}{rrrrrr}
\hline Bayes_MAE & Bayes_MAPE & Bayes_RMSFE & VECX_MAE & VECX_MAPE & VECX_RMSFE \\
\hline 0.087 & 0.044 & 0.087 & 0.005 & 0.003 & 0.005 \\
0.087 & 0.044 & 0.087 & 0.160 & 0.081 & 0.222 \\
0.102 & 0.052 & 0.105 & 0.227 & 0.116 & 0.276 \\
0.100 & 0.051 & 0.102 & 0.300 & 0.153 & 0.353 \\
0.103 & 0.052 & 0.105 & 0.388 & 0.197 & 0.458 \\
0.106 & 0.054 & 0.108 & 0.484 & 0.245 & 0.573 \\
0.127 & 0.065 & 0.138 & 0.586 & 0.297 & 0.697 \\
0.129 & 0.065 & 0.139 & 0.740 & 0.374 & 0.916 \\
0.126 & 0.064 & 0.135 & 0.901 & 0.456 & 1.131 \\
0.157 & 0.079 & 0.189 & 1.061 & 0.534 & 1.333 \\
\hline
\end{tabular}

The results in Tables 5-14 show that the alternative model, namely the Bayesian model is better in terms of forecasting ability than the baseline VECX model, for all the stocks and for almost every forecasting horizon. This implies that the Bayesian model is robust and unveils the effect of the Covid-19 on the marine companies' stocks.

\section{Conclusion}

In the present paper, we examine the major marine companies' stocks, namely the we examine some of the biggest Marine companies' stocks, namely the Dynangas (DLNG), Euronav (EURN), Euroseas (ESEA), Frontline (FRO), Nordic American Tanker (NAT), Scorpio Bulkers (SALT), Sino-Global Shipping (SINO), Teekay Tankers (TNK), Tsakos Energy Navigation (TNP), Torm (TRMD) during the Covid-19 spread and the lockdown measures. In this context, we constructed and employed a Bayesian framework in order to unveil the effect of the Covid-19 pandemic on these stocks. 
Based on our findings, the stocks were affected by the Covid-19 spread and more precisely, the Covid-19 spread provides useful information for the forecasting of these stocks, as shown by the forecasting comparison of the baseline and alternative models, indicated by the forecasting criteria MAE, MAPE and RMSFE in the 22/10 - 29/07 time period of the year 2020. Our findings are robust, since the out-of-sample forecasting accuracy of the alternative model employed, that explicitly incorporate the pandemic induced by the SARS-COV-2 virus, is superior to the baseline model.

The results give credit to the impact of the Covid-19 spread on the Marine Companies, decreasing their revenues and impacting their stocks. This fact unveils the great impact of the Covid-19 on the marine industries worldwide. In an attempt to minimize the spread of the novel coronavirus, most economies and policy makers have taken extreme lockdown measures that adversely affect the overall microeconomic, macroeconomic and financial conditions in a global scale. As a result, the Covid-19 has shifted the investors' preferences away from the marine companies' stocks as the lockdown measures totally suspended the services of these companies. The economic damage was not only limited on the revenues, but also on the stocks of these companies, causing serious problems in the marine industries.

We anticipate our work to be a starting point for more sophisticated models, testing for other factors that could play a significant role in forecasting the various industries. Clearly, future and more extended research on the subject would be of great interest.

\section{References}

Depellegrina Daniel, Bastianini Mauro, Fadini Amedeo, Menegon Stefano (2020), The effects of COVID-19 induced lockdown measures on maritime settings of a coastal region, Science of The Total Environment, Volume 740, 140123.

Johansen Soren, Juselius Katarina (1990), Maximum likelihood estimation and inference on cointegration-with applications to the demand for money, Oxford Bulletin of Economics and Statistics, 52(2): 169-210.

Lee J-W and W. McKibbin (2004), Estimating the Global Economic Costs of SARS In: S. Knobler, A. Mahmoud, S. Lemon, A. Mack, L. Sivitz, and K. Oberholtzer (Editors), Learning from SARS: Preparing for the next Outbreak, The National Academies Press, Washington DC (0-309-09154-3).

Liu Ying, Gayle Albert A., Wilder-Smith Annelies and Rocklöv Joacim (2020), The reproductive number of COVID-19 is higher compared to SARS coronavirus, Journal of travel medicine, 1-4. 
Phillips Peter C. B. and Perron Pierre (1988), Testing for a Unit Root in Time Series Regression, Biometrika, Vol. 75, No. 2, pp. 335-346.

WHO - World health organization (2020), Coronavirus disease 2019 (COVID-19), Situation Report - 51 .

Wilder-Smith Annelies, Chiew Calvin J and Lee Vernon J (2020), Can we contain the COVID-19 outbreak with the same measures as for SARS?, The Lancet infectious diseases, Personal View, pp.1-6, https://doi.org/10.1016/S1473-3099(20)30129-8.

Wooldridge J. M. (2013), Introductory Econometrics: A Modern Approach. 5th ed. Mason. OH: South-Western. 